\title{
Is there a relation between preserved renal function and oncological outcomes in patients undergoing partial nephrectomy for renal cell carcinoma?
}

\author{
Alessandro Veccia, Riccardo Autorino \\ Division of Urology, VCU Health System, Richmond, Virginia, USA \\ Correspondence to: Riccardo Autorino, MD, PhD, FEBU. Division of Urology, VCU Health System, 1200 East Broad st., Richmond, VA, USA. \\ Email: ricautor@gmail.com. \\ Provenance: This is an invited Editorial commissioned by Section Editor Xiao Li (Department of Urology, Jiangsu Cancer Hospital \& Jiangsu \\ Institute of Cancer Research \& Nanjing Medical University Affiliated Cancer Hospital, Nanjing, China). \\ Comment on: Antonelli A, Minervini A, Sandri M, et al. Below Safety Limits, Every Unit of Glomerular Filtration Rate Counts: Assessing the \\ Relationship Between Renal Function and Cancer-specific Mortality in Renal Cell Carcinoma. Eur Urol 2018;74:661-7.
}

Submitted Oct 16, 2018. Accepted for publication Oct 31, 2018.

doi: $10.21037 /$ atm.2018.11.01

View this article at: http://dx.doi.org/10.21037/atm.2018.11.01

Partial nephrectomy (PN) is the gold standard for treatment of small renal masses (1). The spread of this technique is due to equivalent oncological outcomes compared to radical nephrectomy (RN), better kidney function preservation and impact on cardiovascular outcomes (2). However, the hypothesis that the amount of renal function could influence oncological outcomes is still poorly inquired.

In this interesting article, Antonelli et al. (3) evaluated the correlation between glomerular filtration rate (eGFR) and cancer incidence of mortality. The study involved five academic tertiary institutions that sampled retrospectively oncological and functional data of 3,457 patients with cT1a/T1b/T2, N0M0 renal cell carcinoma (RCC) who underwent surgery with different surgical approach. The authors are to be congratulated for their effort to carrying out such analysis. The main finding was the significant correlation of eGFR and the presence of chronic kidney disease (CKD) Stage 3 with cancer specific mortality (CSM).

This interest data confirms the hypothesis of previous nephrological longitudinal population studies which found linear inverse relationship between eGFR and CSM (4-6). Notable data is the identification of a threshold eGFR value $(65 \mathrm{~mL} / \mathrm{min})$ beyond which CSM seems not to be influenced by kidney function. The strength of this analysis is the possibility to assess and adjust for treatment, pathological features and causes of mortality. However, the retrospective nature makes unable to control for selection bias and unmeasurable confounders.

Previous literature strongly remarked how $\mathrm{PN}$ preserves renal function in an elective and imperative setting (7). Nevertheless, local recurrence-free and cancer specific survival rates were slightly inferior in an imperative setting. Paradoxically, the results of this paper are in contrast with those of previous literature.

One of the indications to imperative nephron sparing surgery (NSS) is impaired kidney function. Zabor et al. demonstrated that CKD patients tend to recover renal function after RN, more likely among patients with lower preoperative eGFR (8). Otherwise, Antonelli et al. found that every nephron unit spared influence CSM (3). This means that patients with the risk of decrease of eGFR under threshold level after RN should be considered for PN. These results could be cue for further discussion about appropriate criteria of renal impairment for imperative NSS and could make this surgery indication reconsidered.

Although these study findings are relevant, there are few caveats that should be pointed.

Many would argue that is difficult to explain and hypothesize how eGFR could influence prognosis after NSS for kidney cancer. Confounding bias as the evolution of surgical technique and tumor features could influence CSM.

In summary, evidence supporting NSS are well recognized and this article add a further hint to consider it 
as preferred surgical approach even for larger renal masses. The doubt about correlation between eGFR and CSM after PN still remains but it opens a window on an unexplored field that requires ulterior insights.

\section{Acknowledgements}

None.

\section{Footnote}

Conflicts of Interest: A Veccia is an Italian Society of UrologyAmerican Urological Association research fellow. R Autorino has no conflicts of interest to declare.

\section{References}

1. Ljungberg B, Bensalah K, Canfield S, et al. EAU guidelines on renal cell carcinoma: 2014 update. Eur Urol 2015;67:913-24.

2. Wang Z, Wang G, Xia Q, et al. Partial nephrectomy vs. radical nephrectomy for renal tumors: A meta-analysis of renal function and cardiovascular outcomes. Urol Oncol

Cite this article as: Veccia A, Autorino R. Is there a relation between preserved renal function and oncological outcomes in patients undergoing partial nephrectomy for renal cell carcinoma? Ann Transl Med 2018;6(Suppl 1):S88. doi: 10.21037/ atm.2018.11.01 2016;34:533.e11-9.

3. Antonelli A, Minervini A, Sandri M, et al. Below Safety Limits, Every Unit of Glomerular Filtration Rate Counts: Assessing the Relationship Between Renal Function and Cancer-specific Mortality in Renal Cell Carcinoma. Eur Urol 2018;74:661-7.

4. Christensson A, Savage C, Sjoberg DD, et al. Association of cancer with moderately impaired renal function at baseline in a large, representative, population-based cohort followed for up to 30 years. Int J Cancer 2013;133:1452-8.

5. Lowrance WT, Ordoñez J, Udaltsova N, et al. CKD and the risk of incident cancer. J Am Soc Nephrol 2014;25:2327-34.

6. Weng PH, Hung KY, Huang HL, et al. Cancer-specific mortality in chronic kidney disease: longitudinal follow-up of a large cohort. Clin J Am Soc Nephrol 2011;6:1121-8.

7. Adkins KL, Chang SS, Cookson MS, et al. Partial nephrectomy safely preserves renal function in patients with a solitary kidney. J Urol 2003;169:79-81.

8. Zabor EC, Furberg H, Lee B, et al. Long-Term Renal Function Recovery following Radical Nephrectomy for Kidney Cancer: Results from a Multicenter Confirmatory Study. J Urol 2018;199:921-6. 